\title{
MICROSPOROGÉNESIS DE TRES ESPECIES DEL SUBGÉNERO PASSIFLORA (PASSIFLORA, PASSIFLORACEAE) DE PARAGUAY
}

\author{
Claudia Pereira Sühsner ${ }^{1}$, Ana I. Honfi², María S. Ferrucci ${ }^{3}$ \& Norma Deginani ${ }^{4}$
}

\begin{abstract}
${ }^{1}$ Laboratorio de Análisis de Recursos Vegetales. Departamento de Biología. Facultad de Ciencias Exactas y Naturales - Universidad Nacional de Asunción, Campus Universitario, San Lorenzo, 1039, Central, Paraguay; clauddinha_7@hotmail.com (autor corresponsal).

${ }^{2}$ Laboratorio de Citogenética Vegetal, Programa de Estudios Florísticos y Genética Vegetal, Instituto de Biología Subtropical (UNaM-CONICET), Facultad de Ciencias Exactas, Químicas y Naturales - Universidad Nacional de Misiones, Rivadavia 2370, 3300, Posadas, Misiones, Argentina.

${ }^{3}$ Instituto de Botánica del Nordeste (UNNE-CONICET), Casilla de Correo 209, 3400 Corrientes, Argentina

${ }^{4}$ Instituto de Botánica Darwinion (ANCEFN-CONICET), Labardén 200, Casilla de Correo 22, B1642HYD, San Isidro, Buenos Aires, Argentina.
\end{abstract}

\begin{abstract}
Pereira Sühsner C., A. I. Honfi, M. S. Ferrucci \& N. Deginani. 2016. Microsporogenesis of three species of subgenus Passiflora (Passiflora, Passifloraceae) from Paraguay. Darwiniana, nueva serie 4(1): 45-56.

The aim of the present study was to characterize karyologically species of Passiflora L. from Félix Pérez Cardozo District, Guairá, Paraguay. The chromosome numbers in meiosis $2 n=9$ II for $P$. alata Curtis and $P$. caerulea L. are reported for the first time. Chromosome number for $P$. edulis Sims $(2 n=$ 9II) is also confirmed. Meiotic behaviour in all studied species was normal with regular chromosome segregation. The most frequent chromosome associations were bivalents at diakinesis and metaphase I. Few meiotic irregularities were observed, such as lagging chromosomes and asynchronous phases of meiosis II, however, pollen viability was high, between $78.83 \%$ and $98.6 \%$. The obtained results confirms $x=9$ as the basic chromosome number for subgenus Passiflora. The present study is a relevant contribution to the knowledge on the cytogenetics of species of Passiflora subg Passiflora from Paraguay.
\end{abstract}

Keywords. Meiosis; Passiflora; pollen viability.

Resumen. Pereira Sühsner C., A. I. Honfi, M. S. Ferrucci \& N. Deginani. 2016. Microsporogénesis de tres especies del subgénero Passiflora (Passiflora, Passifloraceae) de Paraguay. Darwiniana, nueva serie 4(1): 45-56.

El presente trabajo tuvo por objeto caracterizar cariológicamente las especies del género Passiflora L., subgénero Passiflora, del Distrito Félix Pérez Cardozo, Guairá, Paraguay. Por primera vez, se da a conocer el número cromosómico en meiosis $2 n=9$ II para $P$. alata Curtis y $P$. caerulea L. También, se confirma el número cromosómico de $2 n=9$ II para $P$. edulis Sims. El comportamiento meiótico en todas las especies estudiadas fue normal, con segregación regular de los cromosomas. La asociación cromosómica frecuentemente encontrada en diacinesis y metafase I fue de bivalentes. Las pocas irregularidades meióticas observadas consistieron en cromosomas rezagados en anafase I y fases asincrónicas en meiosis II, sin embargo, la viabilidad de polen es alta, entre 78,83\% y 98,6\%. Los resultados confirman $x=9$ como número básico para el subgénero Passiflora. Este estudio es una importante contribución al conocimiento sobre la citogenética de las especies de Passiflora subg Passiflora de Paraguay.

Palabras clave. Meiosis; Passiflora; viabilidad del polen. 


\section{INTRODUCCIÓN}

El género Passiflora L. es uno de los más importantes de la familia Passifloraceae por comprender más de 500 especies con alto valor económico para la alimentación, la industria farmacéutica y como ornamental. Habita regiones tropicales y se distribuye principalmente en el continente americano (Baillon, 1886; Killip, 1938; Ulmer \& MacDougal, 2004).

Aproximadamente el $30 \%$ de las especies de Passiflora posee información sobre el número cromosómico (Soares-Scott et al., 2005), se registran especies diploides y poliploides. El número básico de cromosomas para subgéneros y diversos grupos de especies aún resulta controversial. Storey (1950), basándose en estudios realizados en diferentes especies, híbridos interespecíficos y razas poliploides de Passiflora, propuso como número básico $x=3$ ó 6 para el género. Otros autores definen como número básico para Passiflora $x=12$, en lugar de $x=6$, este último es considerado exclusivo del subgénero Delacoba (DC.) Rchb., y es calificado como una autapomorfía del subgénero (De Melo \& Guerra, 2003).

Hansen et al. (2006) relacionaron los clados de Passiflora con el número cromosómico de las especies que los integran, y caracterizaron con el número haploide a los subgéneros Delacoba $x=$ 6, Passiflora $x=9$ y $x=12$ para Astrophea (DC.) Mast. y Deidamioides (Harms) Killip. En general, las especies conocidas cromosómicamente pueden agruparse sobre la base del número gamético de cromosomas $(n)$ en tres grupos, reuniendo subgéneros y especies de la siguiente manera: $i) n$ $=6,12,18$; ii) $n=10$ y iii) $n=9,36$ (De Melo et al., 2001). Estos autores reúnen en el primer grupo citológico, a especies diploides $(2 n=12)$, tetraploides $(2 n=24)$ y hexaploides $(2 n=36)$ con similaridades cariológicas, tales como cariotipos formados por cromosomas metacéntricos y submetacéntricos y núcleo interfásico de tipo areticulado (subg. Astephia Killip, Astrophea, Plectostemma Mast., Pseudomurucuja (Harms) Killip y Psilanthus (DC.) Killip). El segundo grupo reúne 20 especies diploides con $2 n=18$ y $n=9$ II junto a un raro registro de $2 n=72$ cromosomas, cuyos rasgos comunes son el tamaño cromosómico y núcleos de interfase de tipo semireticulado (subg.
Calopathanthus (Harms) Killip, Distephana (Juss.) Killip, Granadillastrum Triana \& Planc., Passiflora, Tacsonia (Juss.) Triana \& Planc. y Tacsonioides (DC.) Killip). Finalmente en la última categoría, se ubica una sola especie (subg. Dysosmia (DC.) Killip) que posee $2 n=20$ pequeños cromosomas, formación de 10 II en meiosis y el núcleo de interfase de tipo areticulado.

Los estudios del género en Paraguay son escasos, constituyendo principalmente estudios de la flora regional, estudios florísticos, o de plantas medicinales (Sandoya, 1994; Céspedes et al., 2008; Pin et al., 2009; Pereira et al., 2009). El informe del Conservatoire et Jardin Botaniques, Ville de Genève (Suiza) y Missouri Botanical Garden, SaintLouis, MO (USA), elaborado por R. Spichiger \& N. Rasolofo (2002), estima que existen 18 especies de Passiflora distribuidas ampliamente por el territorio paraguayo, mientras que en el Catálogo de la Flora del Cono Sur (Zuloaga et al., 2008) se mencionan 23 especies.

El presente trabajo tiene como objetivo analizar la meiosis y la viabilidad del polen de las especies del subgénero Passiflora del género Passiflora L., procedentes del Distrito Félix Pérez Cardozo, Departamento Guairá, Paraguay.

\section{MATERIALES Y MÉTODOS}

Las especies estudiadas (Passiflora alata Curtis, $P$. caerulea L., P. edulis Sims) han sido coleccionadas en el distrito Félix Pérez Cardozo, Dpto. Guairá, Paraguay. Se coleccionaron un total de 46 ejemplares del género, cuyos ejemplares de herbario se depositaron en el Herbario de la Facultad de Ciencias Exactas y Naturales - Universidad Nacional de Asunción (FaCEN - UNA) (Tabla 1).

Para la identificación taxonómica de las especies fueron utilizados los criterios de Killip (1938) y Deginani (1999; 2001). A los fines de elaborar una base de datos citogenéticos útil de las especies de Passiflora, también se constataron las sinonimias.

Para estudiar la microsporogénesis, se obtuvieron anteras inmaduras a partir de botones florales jóvenes fijados directamente en el campo durante las colecciones botánicas. La fijación del material, se realizó con etanol absoluto: ácido láctico glacial 
Tabla 1. Lista de especies de Passiflora estudiadas, de Paraguay, Depto. IV. Municipio Félix Pérez Cardozo; datos de colección y georreferencias.

\begin{tabular}{|c|c|c|}
\hline Especie & Localidad y ejemplar testigo & Georreferencia (UTM) \\
\hline \multirow{2}{*}{ Passiflora alata Curtis * } & Casco urbano. Pe47 (FACEN) & $-25,74194444 /-56,52388889$ \\
\hline & Casco urbano. Pe50 (FACEN) & $-25,74111111 /-56,52583333$ \\
\hline \multirow{17}{*}{ P. caerulea L. * } & Casco urbano. Pel (FACEN) & $-25,74555556 /-56,52222222$ \\
\hline & Casco urbano. Pe2 (FACEN) & $-25,74 /-56,52222222$ \\
\hline & Casco urbano. Pe3 (FACEN) & $-25,745 /-56,52222222$ \\
\hline & Cerrito. Pe4 (FACEN) & $-25,75277778 /-56,53055556$ \\
\hline & Casco Urbano. Pe5 (FACEN) & $-25,74083333 /-56,52222222$ \\
\hline & Casco Urbano. Pe7 (FACEN) & $-25,74416667 /-56,52277778$ \\
\hline & Estación Costa. Pe 9 (FACEN) & $-25,73805556 /-56,51888889$ \\
\hline & Casco Urbano. Pell (FACEN) & $-25,74083333 /-56,52222222$ \\
\hline & Casco Urbano. Pel3 (FACEN) & $-25,745 /-56,52222222$ \\
\hline & Casco Urbano. Pe14 (FACEN) & $-25,745 /-56,52222222$ \\
\hline & Casco Urbano. Pe15 (FACEN) & $-25,745 /-56,52222222$ \\
\hline & Cerrito. Pe16 (FACEN) & $-25,74305556 /-56,5225$ \\
\hline & Casco Urbano. Pe17 (FACEN) & $-25,745 /-56,52222222$ \\
\hline & Cerrito. Pe18 (FACEN) & $-25,75277778 /-56,53055556$ \\
\hline & Casco Urbano. Pe19 (FACEN) & $-25,745 /-56,52222222$ \\
\hline & Cerrito. Pe20 (FACEN) & $-25,74305556 /-56,5225$ \\
\hline & Potrero Melgarejo. Pe21(FACEN) & $-25,74111111 /-56,52722222$ \\
\hline & Casco Urbano. Pe22 (FACEN) & $-25,74666667 /-56,52027778$ \\
\hline & Estación Costa. Pe23 (FACEN) & $-25,75083333 /-56,52027778$ \\
\hline & Estación Costa. Pe24 (FACEN) & $-25,75555556 /-56,51416667$ \\
\hline & Potrero Melgarejo. Pe25 (FACEN) & $-25,73527778 /-56,52777778$ \\
\hline & Cerrito. Pe27 (FACEN) & $-25,75277778 /-56,52027778$ \\
\hline & Cerrito. Pe28 (FACEN) & $-25,75361111 /-56,51916667$ \\
\hline & Cerrito. Pe29 (FACEN) & $-25,75555556 /-56,51916667$ \\
\hline P. caerulea L. * & Cerrito. Pe30 (FACEN) & $-25,74361111 /-56,52888889$ \\
\hline
\end{tabular}




\begin{tabular}{|c|c|c|}
\hline Especie & Localidad y ejemplar testigo & Georreferencia (UTM) \\
\hline & Cocuere Guazu. Pe31 (FACEN) & $-25,76305556 /-56,51138889$ \\
\hline & Cocuere Guazu. Pe36 (FACEN) & $-25,77083333 /-56,50166667$ \\
\hline & Cocuere Guazu. Pe37 (FACEN) & $-25,75833333 /-56,50833333$ \\
\hline & Cocuere Guazu. Pe38 (FACEN) & $-25,77166667 /-56,50222222$ \\
\hline & Cocuere Guazu. Pe39 (FACEN) & $-25,76972222 /-56,50472222$ \\
\hline & Casco Urbano. Pe 40 (FACEN) & $-25,745 /-56,52222222$ \\
\hline & Potrero Melgarejo. Pe41 (FACEN) & $-25,74 /-56,55277778$ \\
\hline & Casco Urbano. Pe42 (FACEN) & $-25,745 /-56,52222222$ \\
\hline & Casco Urbano. Pe43 (FACEN) & $-25,745 /-56,52222222$ \\
\hline & Casco Urbano. Pe44 (FACEN) & $-25,745 /-56,52222222$ \\
\hline & Cocuere Guazu. Pe46 (FACEN) & $-25,76888889 /-56,51611111$ \\
\hline & Cerrito. Pe62 (FACEN) & $-25,74361111 /-56,52888889$ \\
\hline \multirow{7}{*}{ Passiflora edulis Sims. } & Potrero Melgarejo. Pe26 (FACEN) & $-25,74055556 /-56,55277778$ \\
\hline & Casco Urbano. Pe32 (FACEN) & $-25,74555556 /-56,52194444$ \\
\hline & Casco Urbano. Pe33 (FACEN) & $-25,74 /-56,52138889$ \\
\hline & Cocuere Guazu. Pe34 (FACEN) & $-25,7625 /-56,51138889$ \\
\hline & Cocuere Guazu. Pe35 (FACEN) & $-25,76444444 /-56,51194444$ \\
\hline & Potrero Melgarejo. Pe53 (FACEN) & $-25,74111111 /-56,52722222$ \\
\hline & Cocuere Guazu. Pe54 (FACEN) & $-25,77083333 /-56,505$ \\
\hline
\end{tabular}

* Primer estudio meiótico para la especie

en proporción 5:1 (Fernández, 1973). A fin de observar las células madre del polen (CMP), se realizó una incisión transversal de la antera y luego se maceró suavemente en una gota de carmín acético al $2 \%$. Posteriormente se aplastó el material y se observó al microscopio óptico. Los preparados con CMP se hicieron permanentes con solución de Terpentina de Venecia, para ello se agregó una gota de dicha solución en uno de los bordes del cubreobjeto y se facilitó su difusión colocando en el lado opuesto un triángulo de papel de filtro para absorber el colorante. Se dejó secar el medio de montaje al menos por 72 horas.

Los estudios de viabilidad del polen se realiza- ron con la técnica de carmín: glicerina (1:1), se dejó actuar el colorante durante 48 horas y se determinó el porcentaje de granos de polen teñidos sobre un mínimo de 1000. La clasificación del tamaño del grano de polen se basó en el eje de mayor longitud (eje polar o diámetro ecuatorial), de acuerdo al criterio de Erdtman (1966): $<10 \mu \mathrm{m}$ muy pequeño, 10 a $25 \mu \mathrm{m}$ pequeño, 25 a $50 \mu \mathrm{m}$ mediano, 50 a 100 $\mu \mathrm{m}$ grande, 100 a $200 \mu \mathrm{m}$ muy grande, $>200 \mu \mathrm{m}$ gigante.

Las fotomicrografías fueron tomadas con cámara digital MOTICAM (2006), incorporada al microscopio óptico marca OLYMPUS serie CX31 con ocular de 100x. 

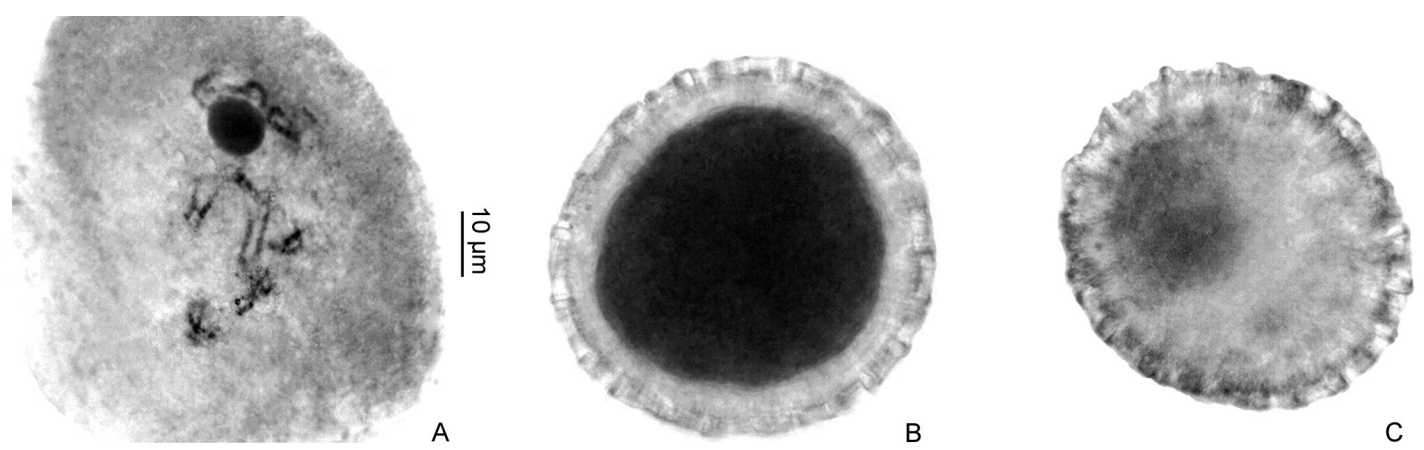

Fig. 1. Microsporogénesis de Passiflora alata. A, CMP en diplotene con 9II. B, Grano de polen coloreado, viable. C, Grano de polen no coloreado, no viable.

Tabla 2. Viabilidad de polen para las especies de Passiflora estudiadas.

\begin{tabular}{|c|c|c|c|c|}
\hline \multirow{2}{*}{ Especie } & \multirow{2}{*}{ Ejemplar } & \multirow{2}{*}{$\begin{array}{c}\text { Viabilidad de Polen } \\
\qquad(\%)\end{array}$} & \multicolumn{2}{|c|}{$\%$ Inviables } \\
\hline & & & Vacíos & Colapsados \\
\hline P. alata & Pe50 & 98,6 & 0,8 & 0,6 \\
\hline \multirow{15}{*}{ P. caerulea } & Pel & 70,30 & 0,20 & 29,50 \\
\hline & $P e 2$ & 99,20 & 0,80 & 0 \\
\hline & $\mathrm{Pe} 3$ & 97,70 & 2,30 & 0 \\
\hline & Pe4 & 97,70 & 2,30 & 0 \\
\hline & $P e 9$ & 53,60 & 0,10 & 46,30 \\
\hline & Pe15 & 98,70 & 1,30 & 0 \\
\hline & $P e 24$ & 96,20 & 3,80 & 0 \\
\hline & $P e 25$ & 63,10 & 36,90 & 0 \\
\hline & Pe36 & 94,00 & 0,50 & 5,50 \\
\hline & Pe38 & 93,80 & 0,20 & 6,00 \\
\hline & Pe4l & 84,20 & 0,50 & 19,30 \\
\hline & Pe42 & 81,70 & 12,70 & 0 \\
\hline & Pe43 & 92,20 & 3,20 & 4,60 \\
\hline & Pe46 & 66,10 & 33,90 & 0 \\
\hline & $\mathbf{x}$ & 84,61 & 7,05 & 8,34 \\
\hline \multirow{3}{*}{ P. edulis } & Pe53 & 95 & 3 & 2 \\
\hline & Pe54 & 97,60 & 1,30 & 1,10 \\
\hline & $\mathbf{x}$ & 96,30 & 2,15 & 1,55 \\
\hline
\end{tabular}



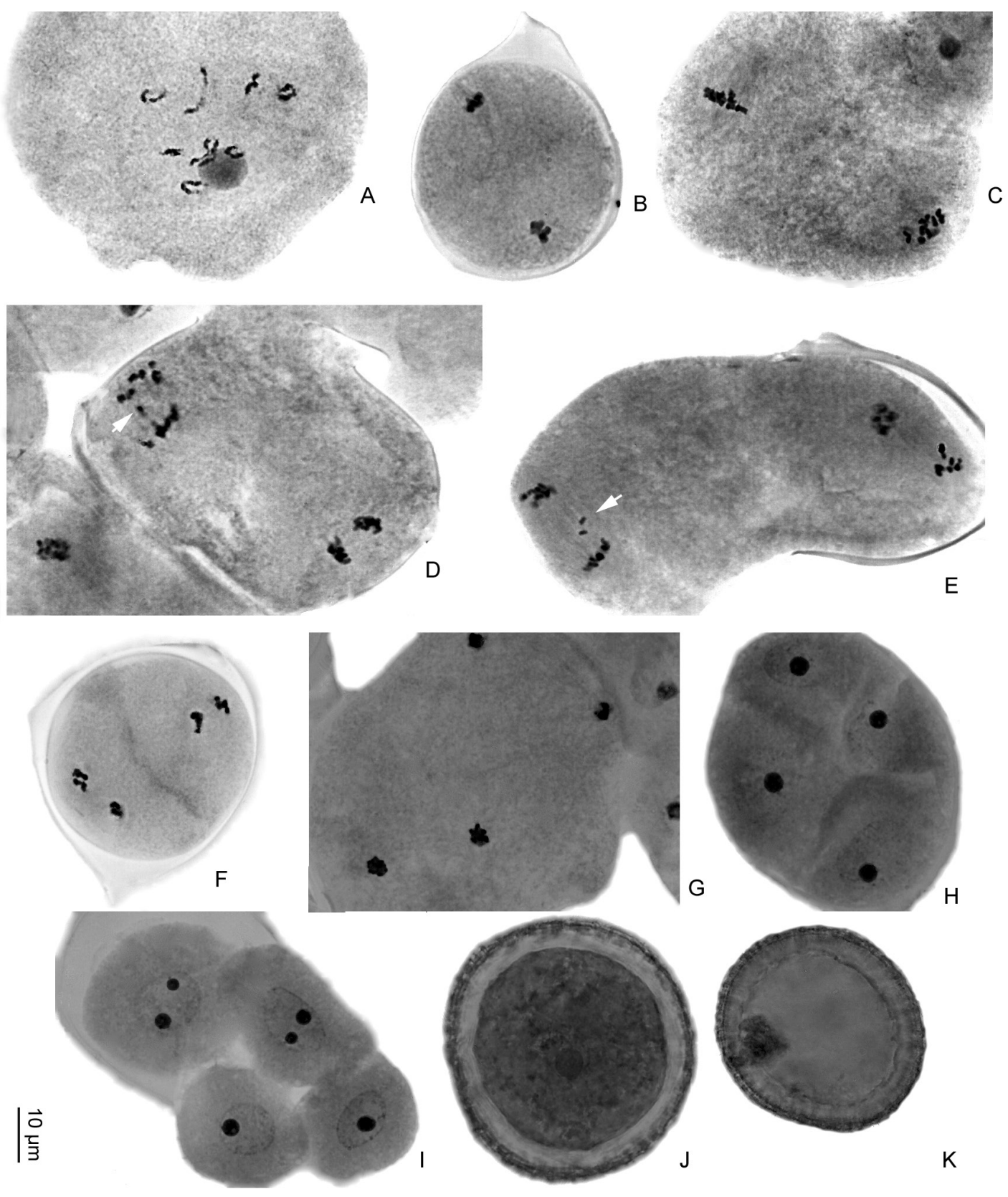

Fig. 2. Microsporogénesis de Passiflora caerulea A, CMP en diplotene, con 9 II. B, CMP en telofase I. C, CMP en metafase II. D, CMP en anafase II (flecha señala un cromosoma rezagado). E, CMP en anafase II avanzada, asincrónica (flecha señala dos cromosomas rezagados). F, CMP en telofase II con primer fragmoplasto formado. G, CMP con los cuatro núcleos en telofase II. H, Tétrade de microsporas reducidas (n). I, Tétrade de microsporas, dos microsporas con dos nucléolos de diferente tamaño rodeados por la membrana nuclear, dos microsporas con sólo uno por núcleo. J, Grano de polen con citoplasma coloreado, viable. K, Grano de polen con citoplasma no coloreado, inviable. 

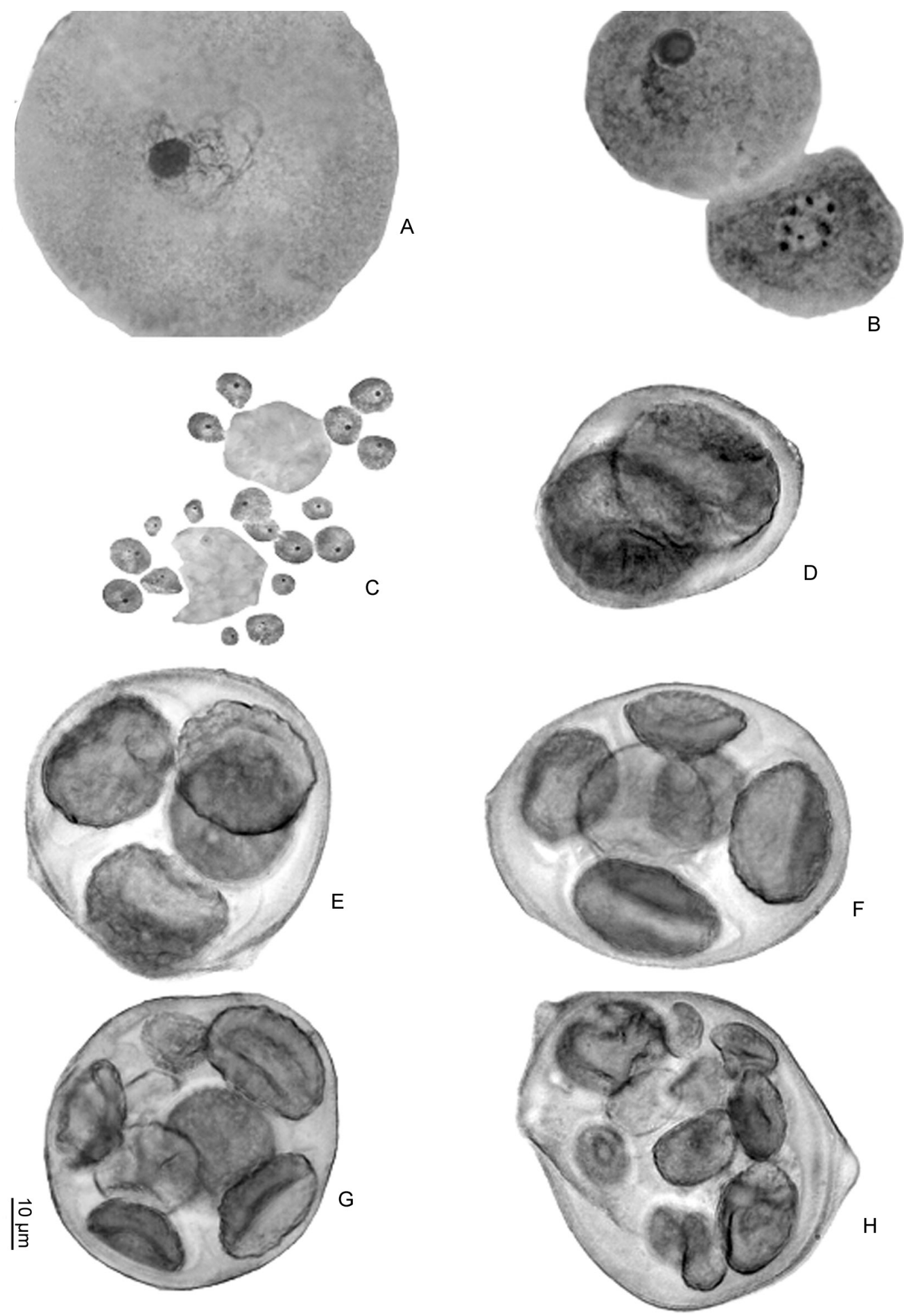

Fig. 3. Microsporogénesis de P. caerulea L. (Pe39). A, CMP en profase temprana. B, Microspora con $n=9$. Un cromosoma ausente en otro plano. C, Políadas con microsporas libres. D, E, Tétrade con cuatro microsporas. F, Políada con seis microsporas. G, Políadas con ocho microsporas. H, Políada con doce microsporas. La barra indica $10 \mu \mathrm{m}$. 


\section{RESULTADOS}

\section{Passiflora alata Curtis.}

Se analizó el comportamiento meiótico de una procedencia de esta especie y se encontró que el nucléolo es persistente hasta metafase I y la única asociación cromosómica encontrada en diacinesis y metafase I han sido 9II en todas las CMP analizadas (Fig. 1A). La meiosis tiene un comportamiento regular y concluye con la formación de cuatro microsporas que darán origen a cuatro granos de polen $(n)$. La viabilidad del polen ha sido $98,6 \%$, los granos inviables son ligeramente más pequeños que los viables (Figs. 1B-C, Tabla 2). Se observaron dos tipos de granos inviables, vacíos y colapsados en promedio de $0,8 \%$ y $0,6 \%$ respectivamente.

\section{Passiflora caerulea L.}

Se analizaron 19 procedencias de esta especie y en todas se encontraron $2 n=9$ II. (Tabla 1 ). La única asociación cromosómica encontrada en diacinesis y metafase I ha sido 9II (Fig. 2A). El comportamiento meiótico ha sido regular en todas las procedencias analizadas (Fig. 2B-C, F). La mayoría de las CMP en anafase I presentan comportamiento cromosómico regular durante la segregación. Ocasionalmente se observaron comportamientos meióticos inusuales, como la presencia de uno o dos pares de cromosomas rezagados (Fig. 2D-E). La presencia de cromosomas rezagados ha sido frecuentemente observada en anafase II. La disposición y orientación de los husos acromáticos durante la meiosis II fue generalmente perpendicular (Fig. 2D) y rara vez se observaron paralelos (Fig. 2E). En algunas CMP en metafase II y anafase II se observó comportamiento asincrónico de los cromosomas, es decir, que los conjuntos cromosómicos de cada huso acromático se encontraban en distintas etapas de la migración. Se observaron cuatro microsporas como producto meiótico (Fig. $2 \mathrm{H}$ ), ocasionalmente se observa una o dos microsporas con dos o tres nucléolos por núcleo (Fig. 2I). La meiosis concluye con la formación de cuatro microsporas genéticamente reducidas ( $n$ ) (Fig. $2 \mathrm{G})$. La viabilidad del polen en promedio ha sido $84,61 \%$, los granos inviables son ligeramente más pequeños que los viables (Figs. 2J-K, Tabla 2). Se observaron dos tipos de granos inviables, vacíos y colapsados con porcentaje promedio de 7,05\% y $8,34 \%$ respectivamente.

Por otro lado, una de las accesiones de P. caerulea (Pe39), presentó una meiosis regular (Fig. 3A-B) y como resultado post-meiótico políadas con microsporas libres, o tétradas o políadas con seis, ocho, diez o doce microsporas (Figs. 3C-H), que probablemente resultan de divisiones mitóticas subsiguientes a la formación de la tétrade de microsporas, cromosómicamente reducidas $(n)$. Dichas políadas están formadas por microsporas (n) y todas rodeadas por calosa (Figs. 3C-H). Los granos de polen son de tamaño mediano, cuyo eje polar oscila entre $25 \mu \mathrm{m}$ y $50 \mu \mathrm{m}$. La viabilidad del polen para esta accesión (Pe39) fue 52,76 \%, y el $47,24 \%$ restante refiere a polen inviable y de tamaño diverso.

\section{Passiflora edulis Sims}

Se analizaron 2 procedencias de esta especie y en ambos casos se encontraron $2 n=9$ II. En diplotene, diacinesis y metafase I se encontraron 9II en todas las células analizadas (Figs. 4A-B). La mayoría de las CMP presentaron comportamiento cromosómico regular durante la segunda división meiótica (Fig. 4C). La orientación del huso en la anafase II fue generalmente perpendicular (Fig. 4D), rara vez en paralelo (Fig. 4E). Se observaron tétradas con cuatro microsporas como producto meiótico (Fig. 4F). El promedio de granos de polen viables fue 96,30 \% (Figs. 4G- I; Tabla 2).

\section{DISCUSIÓN}

Hansen et al. (2006) relacionó el número básico de cromosomas con los cuatro subgéneros: Astrophea, Deidamioides, Delacoba y Passiflora (Feuillet \& MacDougal, 2003). Las tres especies aquí analizadas corresponden al subgénero Passiflora, que se caracteriza por reunir especies con $x=9$.

Se menciona por primera vez el comportamiento meiótico con $n=$ 9II para $P$. caerulea y P. alata; no obstante, Heitz (1926), Nakajima (1931), Simonet \& Miedzyrzecki (1932), Bowden (1940, 1945), De Melo (2001) y Chiapero et al. (2013) reportaron para $P$. caerulea $2 n=18$. De 

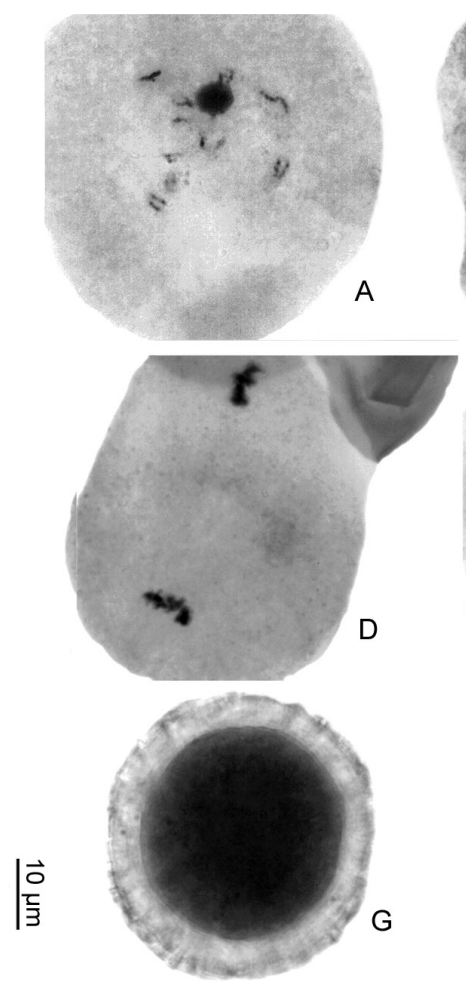
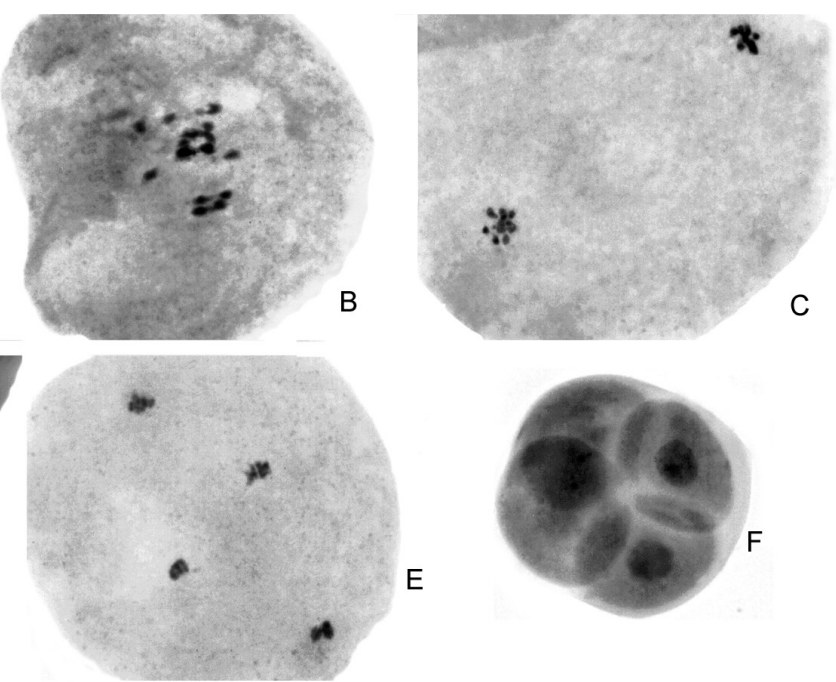

$\mathrm{E}$
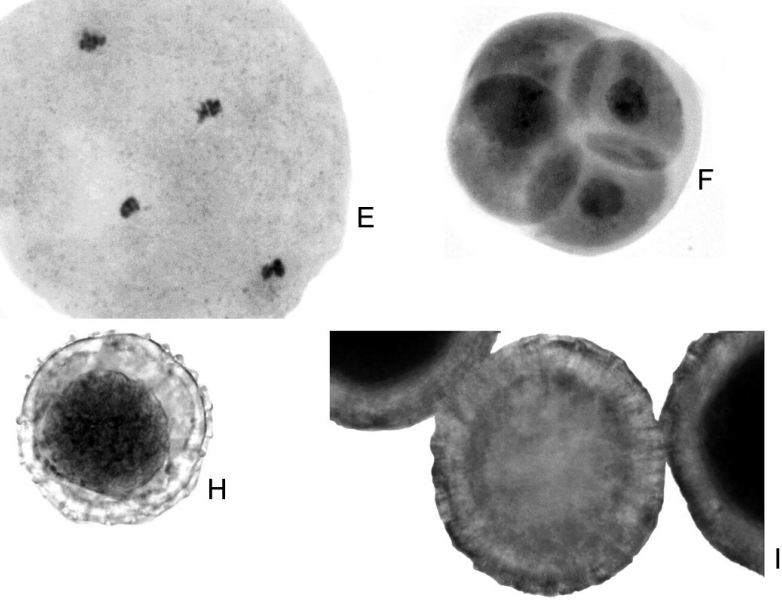

Fig. 4. Microsporogénesis en Passiflora edulis Sims. A, CMP en diplotene, con 9 II. B, CMP en Metafase I, 9 II. C, D, CMP en metafase II. E, CMP en telofase II. Nótese husos perpendiculares en D y paralelos en E. F, Tétrade de microsporas reducidas. G, Grano de polen con citoplasma coloreado, viable. H, Grano de polen con citoplasma colapsado, inviable. I, Grano de polen con citoplasma no coloreado, inviable.

igual manera, para $P$. alata Storey (1950), Guerra (1986), De Melo (2001, 2003) y Sorza (2003) observaron $2 n=18$. El número cromosómico hallado en meiosis de $P$. edulis $2 n=9$ II, coincide con la cita de Gill et al. (1984) y con los reportes de Janaki Ammal (1945), Storey (1950) y Guerra (1986) quienes encontraron $2 n=18$ cromosomas en mitosis.

Todas las especies resultaron diploides con comportamiento regular durante la microsporogénesis y forman granos de polen viables. Estos resultados coinciden con las observaciones de varios autores (De Melo et al., 2001; Souza, 2002; Souza et al., 2003), quienes estudiaron la meiosis de otras especies diploides y donde la asociación cromosómica más frecuentemente observada fueron bivalentes. Diversos trabajos reportaron irre- gularidades durante la meiosis, como la presencia de univalentes, cromosomas rezagados, fases asincrónicas, puentes anafásicos, micronúcleos, citocinesis aberrante y microcitos, apareamiento de cromosomas de tamaños diferentes, asociaciones secundarias de los cromosomas en metafase I, diferentes posiciones del huso acromático formando distintos patrones de tétradas y degeneramiento citoplasmático (Barbosa \& Vieira, 1997a; Olaya, 2002; Souza et al., 2003). Sin embargo, en las especies analizadas el comportamiento fue regular durante la segregación cromosómica en anafase I, y concuerda con lo reportado por Barbosa \& Viera (1997a, b), De Melo et al. (2001) y Souza (2002).

La viabilidad del polen está relacionada con el comportamiento meiótico o eventos post-meióti- 
cos (Barrios, 2005; Souza et al., 2008). Ferreira (1994) considera que las especies de Passiflora con $2 n=18$ presentan una meiosis regular y alta fertilidad, consideración que concuerda con las especies analizadas aquí, que presentaron una viabilidad del polen cuyo rango varía entre $78,83 \%$ y $98,6 \%$.

La formación de políadas en una de las accesiones de $P$. caerulea resulta un dato novedoso en la especie pero ya fueron encontradas en el género. La presencia de esporadas diferentes a tétrades de microsporas, resultantes de la meiosis ha sido observada en $P$. amethystina Mikan, $P$. cincinnata Mast., P. edulis Sims., P. gibertii N. E. Brown, $P$. kermesina Link \& Otto, P. malacophylla Mast., $P$. mucronata Lam., todas con $2 n=18$ cromosomas, P. rubra L. $(2 n=12)$, P. pentagona Mast. y $P$. suberosa L. $(2 n=24)$ (Souza y Santana Pereira, 2011), sin embargo fueron entendidas como resultantes de anomalías en la disposición del huso acromático en meiosis II.

Una de las explicaciones posibles para el origen de las políadas de la accesión P39 podría considerar que en lugar de tétradas de microsporas, se formaron otras esporadas con mayor número de células, sin embargo, no se observaron indicios de comportamiento meiótico irregular que sustenten esta hipótesis. Más probablemente, se trata de un proceso donde las tétradas de cuatro microsporas formadas por meiosis permanecen rodeadas por calosa mientras se dividen por mitosis sucesivas dando lugar a la políada. Además, las políadas observadas en $\mathrm{Pe}$ 39, sugieren que las mitosis post-meiosis no ocurren simultáneamente en todas las microsporas, y por esa razón las mismas contienen $6,8,10$ o 12 microsporas de diverso tamaño. Cada microspora forma un grano de polen de diferente tamaño explicando la heterogeneidad observada en el tamaño del polen.

\section{CONCLUSIONES}

El presente trabajo constituyó el primer estudio citológico para las especies de Passiflora subgénero Passiflora del distrito Pérez Cardozo de Paraguay. Los resultados obtenidos confirman $x=$ 9 como número básico para el subgénero y constituyen un importante aporte al conocimiento so- bre la distribución, taxonomía y evolución de las especies de Passiflora, así como también son de utilidad para el mejoramiento genético, la conservación y manejo de estos recursos genéticos de interés alimenticio y ornamental.

\section{BIBLIOGRAFÍA}

Akamine, E. K. \& D. G. Girolami. 1957. Problems in fruit set in yellow passion fruit. Hawaii Fram Science. 5 (4): 3 - 5.

Baillon, H. 1886. Histoire des plantes, Monographie Des Campanulacees, Cucurbitacees, Loacsacees, Passifloracees \& Begoniacees. Paris: Librairie Hachette \& C. DOI: http:// bibdigital.rjb.csic.es/ing/Libro.php?Libro=6294

Barbosa, L. V.; M. Mondin; C. A. Oliveira; A. P. Souza \& L. V. Vieira. 2007. Cytological behaviour of the somatic hybrids Passiflora edulis f. flavicarpa and P. cincinata. Plant Breeding. 126: 323 - 328. DOI: http://dx.doi.org/10.1111/j.14390523.2007.01362.x

Barbosa, L. V. \& M. L. C. Vieira. 1997a. Meiotic behavior of passion fruit somatic hybrids, Passiflora edulis f. flavicarpa Degener + P. amethystina Mikan. Euphytica 98 (1 - 2): 121 - 127.

Barbosa, L. V. \& M. L. C. Vieira. 1997b. Análise do comportamento meiótico de híbridos somáticos Passiflora edulis $\mathrm{f}$. flavicarpa Degener + P.cincinnata Mast. Revista Brasileira de Genética 20: 88.

Barrios Arango, L. 2005. Estudios de la diversidad de Passifloraceae en los Departamentos de Caldas, Chocó, Nariño, Quindío, Risaralda y Valle del Cauca (Colombia), apoyados en los análisis ecogeográficos, palinológicos y citogenéticos. Tesis (M. Sc.). Palmira, CO: Universidad Nacional de Colombia. $65 \mathrm{p}$.

Bowden, W. M. 1940. The chromosome complement and its evolutionary relationship to cold resistance in the higher plants. Chronica Botanica 6: 123 - 125.

Bowden, W. M. 1945. A list of chromosome numbers in higher plants. II. Menispermaceae to Verbenaceae. American Journal of Botany 32: 191 - 201. DOI: http://dx.doi. org/10.2307/2437510

Cervi, A. 1997. Passifloraceae do Brasil: Estudo do gênero Passiflora L., subgênero Passiflora. Fontqueria 45 (1): 1 - 92.

Céspedes, G.; E. Weber \& G. González. 2008. Especies útiles de la flora paraguaya: Frutos Comestibles. Asunción: AGR Servicios Gráficos.

Chiapero, A. L.; M. L. Las Peñas; M. T. Amela García \& G. Bernardello. 2013. Estudios citogenéticos en especies de Passiflora subgénero Passiflora (Passifloraceae). Boletín de la Sociedad Argentina de Botánica 48 (1): 103- 110. 


\section{S. PEREIRA ET AL. Microsporogénesis de tres especies de Passiflora}

Da Costa, J. 1980. Passifloraceas, em R. Reitz (Ed.) Flora Ilustrada Catarinense, parte 1, fasc. PASS, pp.1-130. Itajaí: Herbario "Barbosa Rodrigues".

De Melo, N. F.; A. C. Cervi \& M. Guerra. 2001. Karyology and cytotaxonomy of the genus Passiflora L. (Passifloraceae). Plant Systematics and Evolution 226: 69 - 84. DOI: http:// dx.doi.org/10.1007/s006060170074

De Melo, N.F. \& M. Guerra. 2003. Variability of the 5 S and 45S rDNA sites in Passiflora L. species with distinct base chromosome numbers. Annals of Botany 92: 309 - 316. DOI: http://dx.doi.org/10.1093/aob/mcg138

Deginani, N. 1999. Passifloraceae L., en L. J. Novara (ed.), Flora del Valle de Lerma (Provincia de Salta, República Argentina). Aportes Botánicos de Salta, Serie Flora 6 (2): 1 - 20.

Deginani, N. 2001. Las especies Argentinas del género Passiflora (Passifloraceae). Darwiniana 39 (1 - 2): 43 - 129.

Deginani, N. \& A. Escobar. 2002. Números cromosómicos de espécies de Passiflora (Passifloraceae). Hickenia 3: 143 - 144.

Erdtman, G. 1966. Pollen morphology and plant taxonomy. Angiosperms. New York: Hafner Publishing Company. 553 p.

Ferreira, F. R. 1994. Germoplasma de Passiflora no Brasil, en A. R. São José (ed.). Maracujá: rodução e mercado. p 24-26 Bahia: Universidade Estadual do Sudoeste da Bahía.

Feuillet, C. \& J. M. Macdougal. 2003. A new infrageneric classification of Passiflora L. (Passifloraceae). Passiflora 13 (2): $34-38$.

Gill, B. S.; S. S. Bir; M. S. Sikdhuu; \& V. K. Singhal. 1984. Chromosome number reports LXXXIV. Taxon 33: 536 - 539.

Guerra, M. 1986. Citogenética de angiospermas coletadas em Pernambuco, I. Revista Brasileira de Genética 9: 21 - 40.

Hansen, A.; L. Gilbert; B. Simpson; S. Downie; A. Cervi \& R. Jansen. 2006. Phylogenetic Relationships and Chromosome Number Evolution in Passiflora. Systematic Botany 31 (1): 138 - 150. DOI: http://dx.doi. org/10.1600/036364406775971769

Heitz, E. 1926. Der nachweis der chromosomen. Vergleichende studien über ihre Zahl, grösse und form im pflanzenreich. I. Zeitschrift für Botanik 18: 625 - 681.

Janaki Ammal, E. K. 1945. Chromosome atlas of cultivated plants. en C. D. Darlington \& E. K. Janaki Ammal (eds.). Londres: George Allen and Unwin Ltd.

Killip, E. P. 1938. The American species of Passifloraceae. Publications of the Field Museum of Natural History, Botanical Series 19: 1 - 613.

Motic China Group. 2006. Motic Images Plus versión 2.0. Software de computadora para microscopia digital.

Nakajima, G. 1931. The chromosome numbers in cultivated and wild Angiosperms. The Botanical Magazine 45: 7 - 11. DOI: http://dx.doi.org/10.15281/jplantres1887.45.7

Olaya Arias, C. A. 2002. Primer estudio de la meiosis en
Passiflora tripartita var. mollissima (Kunth) Holm-Nielsen \& Jorgensen, Passiflora tarminiana Coppens \& Barney, Passiflora mixta y tres de sus híbridos. Tesis de Licenciatura. Universidad de Caldas, Caldas, Colombia.

Pereira, C.; F. González \& B. Benítez. 2009. Evaluación de la morfoanatomía foliar de Passiflora alata Curtis y Passiflora edulis Sims. Steviana 1: 38 - 45.

Pin, A.; G. González; G. Marín; G. Céspedes; S. Cretton; P. Christen \& D. Roguet. 2009. Plantas Medicinales del Jardín Botánico de Asunción. Asunción: AGR Servicios Gráficos.

Sandoya, J. N. 1994. La Cura Natural. Asunción: Editorial Promaster.

Simonet, M. \& C. Miedzyrzecki 1932. Étude caryologique de quelques espèces arborescentes ou sarmenteases d'ornement. Comptes-rendus hebdomadaires des séances et mémoires de la société de biologie 40: 969 - 973.

Soares - Scott, M. D.; M. L. Meletti; L. C. Bernacci \& I. R. Passos. 2005. Citogenética clássica e molecular em passifloras, en: F. G. Faleiro; N. T. V. Junqueira; M. F. Braga (eds.). Maracujá: germoplasma e melhoramento genético. Planaltina: Embrapa Cerrados.

Sorza, M. M.; T. N. S. Pereira; L. C. Silva; D. S. S. Reis \& C. P. Sudre. 2003. Karyotype of six species collected in the state of Rio de Janeiro. Cytologia 68: 165 - 171. DOI: http://dx. doi.org/10.1508/cytologia.68.165

Souza, M.; T. N. Santana; L. Da Cruz Silva; D. Soares \& C. P. Sudre. 2003. Karyotype of six Passiflora species collected in the State of Rio de Janeiro. Cytologia 68: 165 - 171. DOI: http://dx.doi.org/10.1508/cytologia.68.165

Souza, M.; M. M. Souza; T. N. S. Pereira; A. P. Viana; L. C. Silva \& C. P. Sudré. 2004. Pollen viability and fertility in wild and cultivated Passiflora species (Passifloraceae). Beitrage zur Biologie der Pflanzen 73: 359 - 376.

Souza, M. M. 2002. Estudos genômico e reprodutivo em espécies de Passiflora. Tesis doctoral. Universidade Estadual do Norte Fluminense, Campos dos Goytacazes, Brasil.

Souza, M. M.; T. N. Santana \& M. L. Carneiro Vieira. 2008. Cytogenetic Studies in Some Species of Passiflora L. (Passifloraceae): A Review Emphasizing Brazilian Species. Brazilian Archives of Biology and Technology 51 (2): 247 - 258. DOI: http://dx.doi.org/10.1590/S1516-89132008000200003

Spichiger, R \& N. Rasolofo. 2002. Claves y descripciones de las familias y de los géneros de la Flora del Paraguay - Passifloraceae. (En línea). Ginebra: Consultado 22 nov 2009. Disponible en http://www.ville-ge.ch/cbj/fdp/claves/pdf/ passif.pdf.

Storey, W. B. 1950. Chromosome numbers of some species of Passiflora occurring in Hawaii. Pacific Science 4: 7 - 42.

Ulmer, T. \& J. Macdougal. 2004. Passiflora "Passionflowers of the world". Portland: Timber Press Inc. 
Vieira, M. L. \& M. S. Carneiro. 2004. Passiflora spp. Passionfruit. en R. Litz (ed.), Biotechnology of fruit and nut crops. Oxford: CABI Publishing.

Zuloaga, F. O.; O. Morrone \& M. J. Belgrano. 2009. Catálogo de las Plantas Vasculares del Cono Sur (Argentina, Sur de Brasil, Chile, Paraguay y Uruguay) (En línea). Buenos Aires. Consultado 10 oct 2012. 